\title{
Caracterización del banco de semillas de cuatro agroecosistemas de la Frailesca, Chiapas, México
}

\section{Characterization of the seed bank of four agroecosystems in La Frailesca, Chiapas, Mexico}

\begin{abstract}
Carlos Ernesto Aguilar Jiménez ${ }^{1 *}$, Alfredo Tolón Becerra² ${ }^{2}$ Franklin B. Martínez Aguilar ${ }^{3}$, José Manuel Febles González ${ }^{4}$, Héctor Vásquez Solís ${ }^{5}$, Juan Carlos López Hernández ${ }^{6}$.
\end{abstract}

${ }^{1}$ Universidad Autónoma de Chiapas. Facultad de Ciencias Agronómicas. Campus V. Km. 7. Carretera Villaflores-Tuxtla Gutiérrez. C. P. 30470. Villaflores, Chiapas, México.

https://orcid.org/0000-0002-6332-1771

${ }^{2}$ Universidad de Almería, Área de Proyectos de Ingeniería. Carretera Sacramento s/n. La Cañada de San Urbano. 04120. Almería, España.

https://orcid.org/0000-0002-7420-3340

${ }^{3}$ Universidad Autónoma de Chiapas. Facultad de Ciencias Agronómicas. Campus V. Km. 7. Carretera Villaflores-Tuxtla Gutiérrez. C. P. 30470. Villaflores, Chiapas, México.

http://orcid.org/0000-0003-2666-5863

${ }^{4}$ Universidad de La Habana, La Habana, Cuba.

https://orcid.org/0000-0002-9360-1117

${ }^{5}$ Universidad Autónoma de Chiapas. Facultad de Ciencias Agronómicas. Campus V. Km. 7. Carretera Villaflores-Tuxtla Gutiérrez. C. P. 30470. Villaflores, Chiapas, México.

https://orcid.org/0000-0002-3865-9922

${ }^{6}$ Universidad Autónoma de Chiapas. Facultad de Ciencias Agronómicas. Campus V. Km. 7. Carretera Villaflores-Tuxtla Gutiérrez. C. P. 30470. Villaflores, Chiapas, México.

https://orcid.org/0000-0002-5662-8775

$凶$ Autor para correspondencia: ejimenez@unach.mx

\section{Resumen}

El objetivo del trabajo fue caracterizar las arvenses del banco de semillas de agroecosistemas representativos de la región tropical denominada Frailesca, en el estado de Chiapas, México. Los sistemas definidos estuvieron representados por los suelos de vega (márgenes de los ríos), potreros (pastizales), terrenos intermedios para el cultivo de maíz y en sucesión secundaria (acahual). La metodología se fundamentó en la germinación directa, para lo cual se obtuvieron muestras compuestas de suelo a tres profundidades $(0-10,10-20$ y 20-30 cm), las cuales se colocaron en charolas de $50 \times 30 \times 10 \mathrm{~cm}$, ubicándolas en invernadero para evitar su contaminación, proporcionándoles riegos regulares. Cada sistema estuvo representado por tres réplicas, 36 unidades de muestreo totales. Las variables evaluadas fueron número de especies e individuos emergidos, densidad y dominancia relativa, diversidad florística y similitud de comunidades y efectos alelopáticos de las arvenses dominantes. Los resultados indican que los sistemas manejados intensivamente para actividades agrícolas, específicamente terrenos de vega e intermedios para el monocultivo de maíz, presentaron bancos de semillas más dinámicos, lo cual sucede en los primeros $10 \mathrm{~cm}$ de profundidad. Las especies de arvenses dominantes de los bancos evaluados fueron Cyperus rotundus L. y Melampodium divaricatum Rich. La diversidad florística 
determinó índices similares entre los sistemas y profundidades. La mayor similitud entre comunidades se presentó en los agroecosistemas manejados con el cultivo maíz. No se determinaron efectos alelopáticos de las especies dominantes sobre la germinación y crecimiento de los cultivos representativos de la región.

Palabras clave: agroecosistemas, arvenses, reservorio, semillas.

\begin{abstract}
Abstrac
The objective of the work was to determine the dynamics of the weeds of the seed bank of representative agroecosystems of the tropical region, called Frailesca, in the state of Chiapas, Mexico. The defined systems were represented by: the fertile soils (river banks), paddock (grasslands), intermediate lands for the cultivation of corn and in secondary succession (acahual). The methodology was based on direct germination, for which composite soil samples were obtained at three depths $(0-10,10-20$ and 20-30 cm), which were placed in trays of the $50 \times 30 \times 10 \mathrm{~cm}$, placing them in a greenhouse to avoid contamination, providing regular irrigation. Each system was represented by three replicates, 36 total sampling units. The variables evaluated were the number of emerged species and individuals, density and relative dominance, floristic diversity and community similarity, and allelopathic effects of the dominant weeds. The results indicate that the systems intensively managed for agricultural activities, specifically fertile and intermediate lands for the monocultivation of corn, presented more dynamic seed banks, which occurs in the first $10 \mathrm{~cm}$ of depth. The dominant weed species from the evaluated banks were Cyperus rotundus L. and Melampodium divaricatum Rich. Floristic diversity determined similar indices between systems and depths. The greatest similarity between communities was found among the agroecosystems managed for the culture of corn. No allelopathic effects of the dominant species on the germination and growth of the representative crops in the region were determined.
\end{abstract}

Keywords: agroecosystems, weed, reservoir, seeds.

\section{Introducción}

Los agroecosistemas representan sistemas naturales modificados por el hombre con el propósito de producir alimento (Sans, 2007). La intensidad y forma de manejo determinan la dinámica de los factores ecológicos y biológicos que en ellos suceden (Gutiérrez et al., 2008). El crecimiento poblacional ha originado una mayor presión de uso de los recursos naturales en la agricultura. Los primeros sistemas mesoamericanos se caracterizaron por utilizar principios de agricultura itinerante, permitiendo con esto la recuperación temporal de los procesos ecológicos. La demanda de alimentos exigió incorporar nuevas áreas a la agricultura, así como el uso de formas intensivas de producción. El uso de la tecnología supuso la introducción de insumos de síntesis artificial, lo que incrementó los efectos antropogénicos sobre los recursos naturales usados en la agricultura (Gliesssman, 2002). Esta estrategia modificó la dinámica de las malezas, las cuales han coevolucionado con su ambiente.

La dispersión de las semillas es uno de los caracteres funcionales de las especies vegetales, son dispersadas por el viento (anemocoria), el agua (hidrocoria), los animales (epizoocoria), al ser ingeridas por los animales (endozoocoria), por la gravedad (barocoria), por los mecanismos propios de la especie (autocoria), o la combinación de estos agentes (Bedoya-Patiño et al., 2010). El mecanismo de dispersión que utilizan las plantas superiores está determinado por el tipo de vida de la especie; así, las plantas herbáceas se dispersan fundamentalmente por zoocoria y autocoria (Arbeláez \& ParradoRosselli, 2005).

El hombre es uno de los agentes más importantes para la dispersión de semillas. Sus migraciones han llevado consigo a todas partes valiosas plantas de cultivo, así como, inadvertidamente, algunas de las arvenses no deseables. El tamaño de una población ecológica está determinado por el flujo de semillas en su hábitat, que comprende tres fases: maduración de las semillas, traslado de estas desde su progenitor hasta el área en donde caen y movilidad en el reservorio de propágulos en el suelo (banco de semillas) (Granados et al., 2001). Estas etapas se ven afectadas por diferentes factores y la interacción con ellos da como resultado el patrón de dispersión 
de la población. La diseminación es un fenómeno precursor del establecimiento de poblaciones y tiene lugar cuando los frutos y/o semillas, una vez que han sido diseminados, germinan con la finalidad de que los individuos colonicen un nuevo territorio (Cubiña \& Aide, 2001).

Las semillas de las plantas superiores podrán iniciar la colonización del ecosistema una vez que encuentren condiciones ecológicas apropiadas, fundamentalmente humedad y luz. Cuando no existen las condiciones adecuadas para la germinación, estas permanecen en latencia, la cual es considerada un periodo de interrupción de crecimiento $\mathrm{y}$ disminución del metabolismo durante el ciclo vital, es una estrategia adaptativa de supervivencia que se presenta en gran parte de los seres vivientes frente a condiciones ambientales desfavorables (De Sousa et al., 2006). En las plantas superiores puede existir latencia o interrupción de crecimiento en el tejido meristemático, así como en las semillas (Fenner \& Thompson, 2005).

En una comunidad vegetal el banco de semillas es el conjunto de semillas que presentan potencial regenerativo en las comunidades vegetales (Moscoso \& Diez, 2005). La formación del banco de semillas del suelo se inicia con la dispersión y finaliza con la germinación o muerte de las semillas (Walck, 2005). La presencia de diferentes tipos de bancos de semillas permite tener un amplio criterio sobre algunos mecanismos que presentan las especies para coexistir en comunidades perennes, la gran diversidad de bancos indica una variación en la forma, intensidad, distribución estacional y formas de perturbación (Cantillo et al., 2008).

Thompson \& Grime (1979) clasifican a los bancos de semillas del suelo de acuerdo con la viabilidad delas semillas en transitorios y persistentes; sin embargo, esta forma no aporta elementos acerca de la longevidad, ni sobre las posibles prácticas que establezcan manejos adecuados para la restauración de los ambientes. Una versión modificada de esta clasificación fue planteada por Bakker (1989), quien propone tres tipos. Transitorios: bancos cuyas especies persisten en el suelo menos de un año, Persistentes de corto plazo: bancos con semillas de especies que persisten en el suelo por lo menos un año y hasta 5 años, y Persistentes de largo plazo: bancos con semillas de especies que persisten en el suelo por lo menos 5 años. Besnier (1989) menciona dos tipos de distribución de las semillas en el banco del suelo, distribución espacial y distribución vertical, así mismo señala que en general en los terrenos no labrados, las profundidades máximas alcanzadas por las semillas son del orden de $\operatorname{los} 30-35 \mathrm{~cm}$.

La vegetación de un lugar está formada por un componente real y un componente potencial, la primera representada por los individuos de las especies presentes en el área y la segunda por las semillas y propágalos existentes en el suelo. Algunos de los beneficios del estudio del banco de semillas son: determinar la productividad ecológica de los sistemas, el papel fundamental que juegan en la recuperación de áreas fuertemente perturbadas, comprender la dinámica de las poblaciones para el establecimiento de plantas, y en comunidades vegetales anuales agrícolas ayuda a planificar las estrategias de manejo de la flora arvense que compite con las plantas cultivadas (De Souza et al., 2006).

La presente investigación se realizó con el objetivo de caracterizar la flora arvense en el banco de semillas de cuatro sistemas de manejo tradicional, terrenos bajos e intermedios cultivados con maíz, pastizales y suelos en barbecho (descanso), de la Frailesca, Chiapas.

\section{Materiales y métodos}

\subsection{Localización del área de estudio}

El experimento se realizó en las instalaciones de la Facultad de Ciencias Agronómicas, Campus V, de la Universidad Autónoma de Chiapas, municipio de Villaflores, Chiapas, México, localizada en el paralelo $16^{\circ} 15^{\prime} 13.9^{\prime \prime}$ de latitud norte y meridiano $93^{\circ} 15^{\prime}$, 14.2" longitud oeste. La altitud es de $610 \mathrm{msnm}$. De acuerdo con Köppen, modificado por García (1987), el clima que prevalece es el cálido-subhúmedo $\mathrm{AW}_{1}$ (W") (i) g con una temperatura media anual de $22{ }^{\circ} \mathrm{C}$ y una precipitación pluvial media anual de $1.200 \mathrm{~mm}$.

\subsection{Diseño del experimento}

Para caracterizar la dinámica del banco de semillas se definieron cuatro sistemas representativos del uso del suelo en la región Frailesca (Tabla 1). Se utilizó el método de germinación directa, consistente en lograr muestras compuestas de suelo, obteniendo quince submuestras por cada sistema, las cuales se colocaron en charolas $(50 \times 30 \times 10 \mathrm{~cm})$, mismas que se ubicaron en invernadero para evitar su contaminación, proporcionándoles riegos regulares. Cada sistema de manejo estuvo representado con 
tres profundidades $(0-10,10-20$ y $20-30 \mathrm{~cm})$, esto proporcionó 12 tratamientos, cuyas tres repeticiones otorgaron un total de 36 unidades de muestreo. Las muestras de suelo de los diferentes tratamientos se enviaron al laboratorio de Edafología del Colegio de Postgraduados, Campus Montecillos, localizado en el estado de México, para determinar los contenidos de N, P y K, así como su textura.

Tabla 1. Tratamientos definidos para caracterizar el banco de semillas

\begin{tabular}{ll}
\hline \multicolumn{1}{c}{ Sistema } & \multicolumn{1}{c}{ Características } \\
\hline Terreno de vega & Suelos planos aluviales localizados en las márgenes de los ríos. \\
Terreno intermedio de cultivo de maíz & $\begin{array}{l}\text { Suelos semiplanos o de pendientes ligeras (menores de 20\%) usados para el monocultivo } \\
\text { de maíz. }\end{array}$ \\
Potrero o pastizal & $\begin{array}{l}\text { Suelos semiplanos o de pendientes ligeras (menores de 20 \%) usados para el pastoreo } \\
\text { de ganado bovino. }\end{array}$ \\
Acahual & $\begin{array}{l}\text { Suelos semiplanos o de pendientes ligeras (menores de 20 \%) destinados al descanso en } \\
\text { sucesión secundaria de selva media perennifolia. }\end{array}$ \\
\hline
\end{tabular}

\subsection{Variables evaluadas}

Las variables estudiadas en el banco de semillas se midieron en dos tiempos, la primera en muestreos realizados cada 15 días, donde se cuantificó en 10 oportunidades el número de plantas emergidas. El segundo momento, fue al final del experimento (150 días) en donde se determinaron las variables: número de especies y número de individuos, la densidad y dominancia relativa de las principales especies emergidas, la diversidad florística, biomasa total verde y seca, el índice de similitud y se evaluó el potencial alelopático de las arvenses dominantes en los sistemas de manejo intensivo. Las especies emergidas se clasificaron en hoja angosta y ancha, las primeras estuvieron representadas por las poáceas y ciperáceas (denominadas localmente zacates), mientras que las segundas fueron todas las demás (llamadas monte). Para estimar la diversidad se empleó el modelo de Shannon-Wiener (1949) y finalmente se utilizó el índice de Sørensen (1957) para estimar la analogía entre comunidades.

\subsection{Prueba de alelopatía}

Para realizar la prueba de alelopatía se utilizó la metodología propuesta por Gliesssman (2002), con este fin se colectaron las especies más representativas emergidas en los agroecosistemas, que fueron coquillo (Cyperus rotundus L.) y flor amarilla (Melampodioum divaricatum Rich.). De las dos especies previamente colectadas y secadas a la sombra mínimamente durante 72 horas, se pesaron $2,5 \mathrm{~g}$ y $5 \mathrm{~g}$ de cada arvense, colocándose en vasos de precipitados con $100 \mathrm{ml}$ de agua destilada, teniendo así la proporción de 2,5 y $5 \%$ respectivamente, remojándose por un tiempo de dos horas, para provocar la liberación de sustancias alelopáticas, pasado el tiempo, se agregó $10 \mathrm{ml}$ de la solución a cada caja Petri. Para el testigo simplemente se aplicó agua destilada.

En las cajas Petri se experimentó con tres especies cultivadas (maíz, fríjol y calabaza). El procedimiento de preparación de estas consistió en agregar $45 \mathrm{~g}$ de arena, previamente lavada con agua destilada, colocando papel higiénico en la superficie; para distribuir 10 semillas de cada especie indicada con el hipocotilo hacia adentro en cada recipiente utilizado. Las dos especies de arvenses, por tres concentraciones (2,5 y 5,0\%, además del testigo) y tres especies cultivadas (maíz, fríjol y calabaza), originando un total de 18 tratamientos, mismos que estuvieron representados con tres réplicas, para obtener un total de 54 unidades de muestreo. Se sellaron las cajas cuidadosamente y se colocaron ordenadas a la oscuridad, por un lapso de 5 días, transcurrido el tiempo se contó el número de semillas emergidas por especie, así como la longitud radicular de cada individuo emergido.

\subsection{Análisis de la información}

Para conocer las diferencias estadísticas, se realizó el análisis de varianza bajo un diseño 
de clasificación simple, utilizando el paquete de análisis estadístico SPSS, y para comprobar el efecto entre los sistemas se corrieron pruebas de medias, utilizando Tukey al $95 \%$ de probabilidad.

\section{Resultados y discusión}

\subsection{Análisis físico-químico de los suelos}

En la Tabla 2 se muestran los resultados del análisis físico-químico de los suelos de los sistemas estudiados, identificándose texturas migajones con variaciones en su contenido de arena, limo y arcilla, resultados típicos de la región de estudio, donde la dinámica tropical promueve los procesos de pedogé- nesis de una forma acelerada. En cuanto al contenido de nitrógeno se observa una mayor disponibilidad en el método de ecosistema natural (acahual), seguido por el sistema de pastizal (potrero), mientras que los sistemas de vega y terrenos intermedios mostraron determinaciones menores; así mismo, se puede observar una correlación positiva entre la profundidad y las cantidades determinadas, encontrándose las mayores determinaciones en las primeras capas de suelo, disminuyendo a mayores profundidades, resultados similares son reportados por López et al. (2018) al caracterizar los suelos para cultivo de maíz en el mismo territorio. Para fósforo, las mayores determinaciones se establecieron en los sistemas de terrenos de vega y potrero, disminuyendo las determinaciones a mayor profundidad.

Tabla 2. Análisis físico-químico del suelo de cuatro sistemas de estudio en la Frailesca, Chiapas, México.

\begin{tabular}{|c|c|c|c|c|c|}
\hline Sistema & $\begin{array}{l}\text { Profundidad } \\
\text { (cm) }\end{array}$ & Textura & $\begin{array}{l}N \\
(\%)\end{array}$ & $\begin{array}{c}\mathbf{P} \\
(\mathbf{p p m})\end{array}$ & $\begin{array}{c}\mathrm{K} \\
\text { (ppm) }\end{array}$ \\
\hline \multirow{3}{*}{ Acahual } & $0-10$ & & 0,22 & 10 & 0,5 \\
\hline & $10-20$ & Migajón arenoso & 0,25 & 2 & 0,2 \\
\hline & $20-30$ & & 0,08 & 1 & 0,2 \\
\hline \multirow{3}{*}{ Vega } & $0-10$ & & 0,12 & 16 & 0,2 \\
\hline & $10-20$ & Migajón limoso & 0,06 & 5 & 0,1 \\
\hline & $20-30$ & & 0,08 & 8 & 0,3 \\
\hline \multirow{3}{*}{ Maíz } & $0-10$ & & 0,08 & 13 & 0,4 \\
\hline & $10-20$ & Migajón arcillo arenoso & 0,06 & 2 & 0,3 \\
\hline & $20-30$ & & 0,03 & 1 & 0,3 \\
\hline \multirow{3}{*}{ Potrero } & $0-10$ & & 0,16 & 15 & 0,6 \\
\hline & $10-20$ & Migajón arcilloso & 0,13 & 10 & 0,5 \\
\hline & $20-30$ & & 0,08 & 5 & 0,4 \\
\hline
\end{tabular}

En los sistemas de acahual y potrero se encontraron las mayores determinaciones de potasio en las primeras capas del suelo. De forma general, el sistema de no cultivo presentó las mejores determinaciones de elementos mayores, lo cual es producto de la incorporación sistemática de residuos orgánicos de la vegetación en sucesión secundaria (López-Báez et al., 2019). De los tres sistemas manejados, el pastizal indicó tener una fertilidad media, debido al manejo sistemático con herbáceas y a la incorporación de residuos orgánicos de los bovinos que pastorean en él (Apráez et al., 2007); el sistema de cultivo de maíz en terrenos intermedios presenta las determinaciones más bajas, debido a su manejo sistemático de forma convencional y con el monocultivo de maíz (Martínez et al., 2020), lo mismo sucede en el sistema de maíz en terrenos de vega, pero al ser suelos que reciben el aluvión de las partes más altas, estos presentan indicadores de mejor fertilidad química.

López et al. (2019) concluyen que la fertilidad marginal de los suelos cultivados con maíz en la municipalidad de Villaflores y Chiapas, son producto del uso del monocultivo, de la baja incorporación de materia orgánica, de los altos índice de acidez, así como de las deficiencias sistemáticas de los nutrimentos básicos y de una afectación sobre las propiedades físicas de los suelos. Sin embargo, es oportuno mencionar que la calidad de los suelos no tiene influencia en la germinación de las semillas, ya que estás romperán su latencia cuando encuentren las condiciones adecuadas, fundamentalmente de luz y humedad. 


\subsection{Plantas emergidas en los agroecosistemas}

En cuanto a las plantas emergidas en el banco de semillas, las especies de hoja ancha emergieron en mayor cantidad en el sistema de terreno de vega, seguidos por el terreno intermedio de maíz (Tabla 3 y Figura 1), está dinámica es producto del manejo de los sistemas, en donde al ser suelos cultivados con monocultivo de maíz, las especies con mayor área foliar (hoja ancha), tendrán mayor capacidad de captar la radiación solar en medio de las plantas cultivadas (León \& Agüero, 2001). Las especies de hoja angosta emergieron en mayor cantidad en el sistema de vega y pastizal (Tabla 4 y Figura 2), en el primer caso es producto del aluvión recibido por las partes más altas, y en el segundo es producto del manejo con pastos para la ganadería, mismo que es reincorporado por los rumiantes a través de las excretas. Yu et al. (2007) concluyen que las especies con semillas persistentes y redondas son más comunes en los ecosistemas manejados.

Tabla 3. Especies de hoja ancha más comunes en los bancos de semilla

\begin{tabular}{|c|c|c|c|c|}
\hline Sistema & $\begin{array}{l}\text { Profundidad } \\
\text { (cm) }\end{array}$ & Especies & $\begin{array}{c}\text { Densidad } \\
\text { Relativa } \\
(\%)\end{array}$ & $\begin{array}{c}\text { Dominancia } \\
\text { Relativa } \\
(\%)\end{array}$ \\
\hline \multirow{3}{*}{ Terreno de vega } & $0-10$ & Melampodium divariucatum & 6,99 & 18,05 \\
\hline & $10-20$ & Melampodium divariucatum & 5,87 & 33,99 \\
\hline & $20-30$ & ----- & 0 & 0 \\
\hline \multirow{5}{*}{$\begin{array}{l}\text { Terreno } \\
\text { intermedio de } \\
\text { cultivo de maíz }\end{array}$} & \multirow{2}{*}{$0-10$} & Melampodium divariucatum & 12,93 & 39,07 \\
\hline & & Aldama dentata Llave \& Lex & 11,45 & 20,37 \\
\hline & \multirow{2}{*}{$10-20$} & Amaranthus retroflesxus & 9,79 & 4,63 \\
\hline & & Sp no identificada & 38,8 & 26,5 \\
\hline & $20-30$ & ----- & 0 & 0 \\
\hline \multirow{3}{*}{$\begin{array}{l}\text { Potrero o } \\
\text { pastizal }\end{array}$} & $0-10$ & Althaea spp & 2,54 & 14,56 \\
\hline & $10-20$ & Sp no identificada & 48,12 & 38,72 \\
\hline & $20-30$ & Sp no identificada & 26,05 & 27,97 \\
\hline \multirow{4}{*}{ Acahual } & \multirow{2}{*}{$0-10$} & Aldama dentata Llave \& Lex & 21,17 & 41,05 \\
\hline & & Euphorbia heterophylla & 20,98 & 16,31 \\
\hline & $10-20$ & Aldama dentata Llave \& Lex & 13,16 & 34,94 \\
\hline & $20-30$ & ----- & 0 & 0 \\
\hline
\end{tabular}

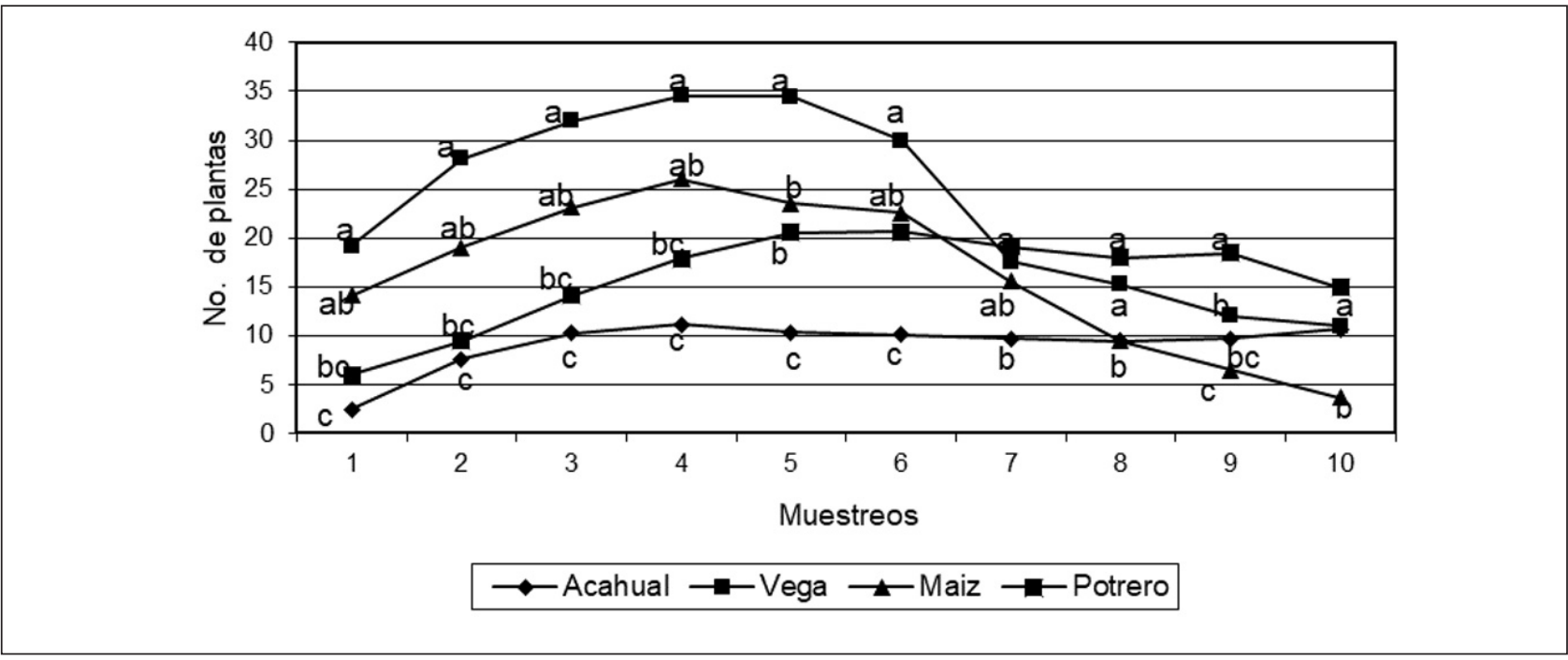

Figura 1. Plantas de hoja ancha emergidas en el banco de semillas. 


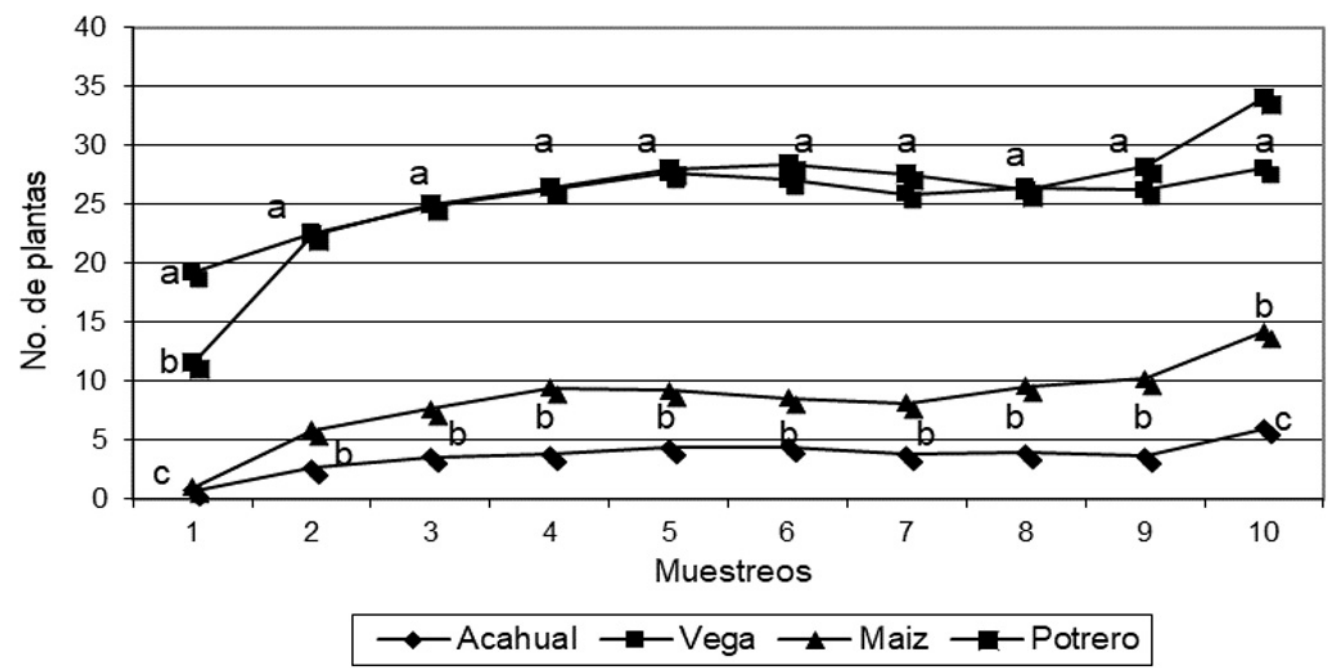

Figura 2. Plantas de hoja angosta emergidas en el banco de semillas.

Tabla 4. Especies de hoja angosta más comunes en los bancos de semilla

\begin{tabular}{|c|c|c|c|c|}
\hline Sistema & $\begin{array}{l}\text { Profundidad } \\
\text { (cm) }\end{array}$ & Especies & $\begin{array}{c}\text { Densidad } \\
\text { Relativa } \\
(\%)\end{array}$ & $\begin{array}{c}\text { Dominancia Relativa } \\
(\%)\end{array}$ \\
\hline \multirow{5}{*}{$\begin{array}{l}\text { Terreno de } \\
\text { vega }\end{array}$} & \multirow{3}{*}{$0-10$} & Cyperus rorundus $\mathrm{L}$. & 40,83 & 20,03 \\
\hline & & Cynodon dactylon (L.) & 16,75 & 15,56 \\
\hline & & Cynodon plectostachius & 5,59 & 6,80 \\
\hline & $10-20$ & Cyperus rorundus $\mathrm{L}$. & 54,45 & 63,22 \\
\hline & $20-30$ & Cyperus rorundus $\mathrm{L}$. & 53,05 & 32,54 \\
\hline \multirow{4}{*}{$\begin{array}{c}\text { Terreno } \\
\text { intermedio de } \\
\text { cultivo de maíz }\end{array}$} & \multirow{2}{*}{$0-10$} & Cenchrus sp & 32,44 & 15,64 \\
\hline & & Cyperus rorundus $\mathrm{L}$. & 24,45 & 17,08 \\
\hline & $10-20$ & ----- & 0 & 0 \\
\hline & $20-30$ & ----- & 0 & 0 \\
\hline \multirow{6}{*}{$\begin{array}{l}\text { Potrero o } \\
\text { pastizal }\end{array}$} & \multirow{4}{*}{$0-10$} & Cyperus rorundus $\mathrm{L}$. & 10,84 & 27,26 \\
\hline & & Sporobulos airoides (Torr.) Torr & 14,78 & 2,33 \\
\hline & & Cynodon plectostachius & 14,78 & 2,33 \\
\hline & & Sorghum helepense & 36,16 & 32,54 \\
\hline & $10-20$ & Cyperus rorundus $\mathrm{L}$. & 19,2 & 6,56 \\
\hline & $20-30$ & Sorghum helepense & 18,87 & 37,2 \\
\hline \multirow{4}{*}{ Acahual } & $0-10$ & Cyperus rorundus $\mathrm{L}$. & 8,79 & 0,26 \\
\hline & $10-20$ & Cyperus rorundus $\mathrm{L}$. & 32,11 & 5,55 \\
\hline & \multirow[t]{2}{*}{$20-30$} & Sporobulos airoides (Torr.) Torr & 43,59 & 69,00 \\
\hline & & Cyperus rorundus $\mathrm{L}$. & 18,33 & 27,52 \\
\hline
\end{tabular}


Finalmente, las especies totales (especies de hoja ancha y angosta) se cuantificaron en mayor medida en el sistema de terreno de vega, seguido por el pastizal, terrenos intermedios de maíz, y por último el terreno en sucesión secundaria (acahual) (Figura 3). La mayor emergencia de especímenes en los terrenos de vega (suelos fluvisoles), obedece a que en ellos se deposita sistemáticamente el aluvión de las partes más altas del territorio, lo que origina una mayor fertilidad del suelo, pero también una mayor dinámica biológica, lo cual se refleja en la diversidad de especies emergidas como flora arvense (Álvarez-Aquino et al., 2005).

La importancia de conocer la dinámica de los bancos de semillas del suelo obedece a que este es un componente importante en la dinámica vegetal y una estrategia de sobrevivencia de las especies a largo plazo, además, cumple un papel fundamental en la recuperación de áreas que han sufrido drásticos procesos de perturbación, siendo estos fundamentales para el mantenimiento de la diversidad florística y la sustentabilidad social y ecológica de los ecosistemas naturales y perturbados (De Souza et al., 2006). Las especies vegetales presentes en cualquier ecosistema natural o modificado requieren de algunos mecanismos, destacándose: la dispersión continua o estacional de las semillas (lluvia de semillas), la acumulación de las semillas en el suelo (banco de semillas), la acumulación de plántulas (banco de plántulas), así como la acumulación de renuevos y el rebrote de estructuras dañadas (Bedoya-Patiño et al., 2010). La dispersión y el banco de semillas son los que determinan en mayor medida el tipo de vegetación que coloniza los ecosistemas.

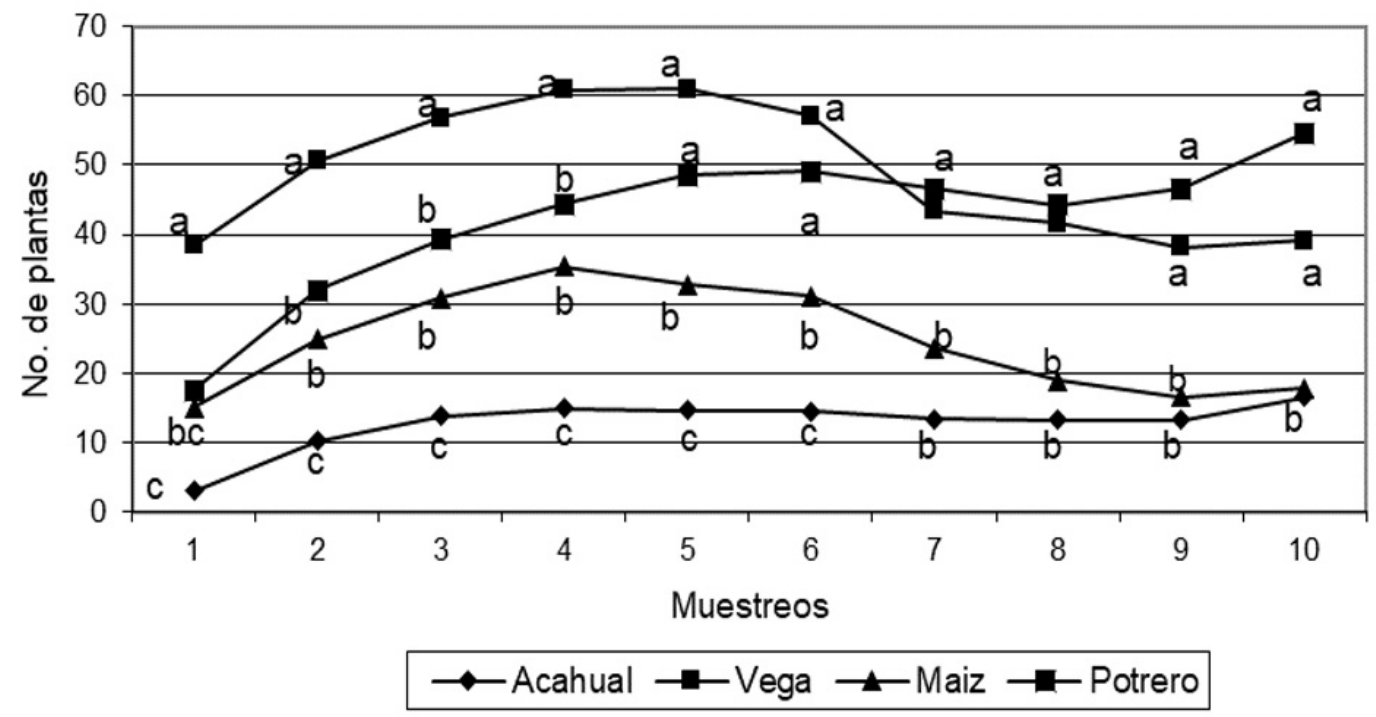

Figura 3. Plantas totales emergidas en el banco de semillas.

El banco de semillas del suelo se compone de semillas aletargadas, semillas en reposo, y de forma continua reciben los estímulos necesarios para romper el letargo y pasan a la situación de reposo, de la que salen para germinar, aunque no todas las semillas que germinan logran su supervivencia; así mismo, el reservorio se enriquece periódica o continuamente, según las circunstancias de dispersión, y también se empobrece continuamente con las semillas que desaparecen o mueren y con las que germinan (Piudo \& Cavero, 2005). Un factor importante a tener en cuenta cuando se evalúa la dinámica de los bancos de semillas de diferentes sistemas es que estas pueden llegar a desaparecer por la depredación llevada a cabo por las aves, mamíferos e insectos (hormigas, principalmente) cuando están en la hojarasca o superficie del suelo, mientras que para las que están enterradas la depredación se reduce mucho, pero algunas semillas pueden sufrir ataques por toda clase de animales, incluso nematodos, así mismo, las semillas enterradas perecen por la pérdida de viabilidad o por ataques de hongos (Price \& Joyner, 1997). 


\subsection{Plantas emergidas en las diferentes profundidades}

En cuanto a la emergencia de las diferentes especies en las tres profundidades estudiadas durante los 10 muestreos realizados, se observa en la Figura 4 una superioridad estadística de mayor presencia de especímenes en los primeros 10 centímetros. Esto nos señala que, en los bancos de semillas de las regiones tropicales, la mayor presencia de los órganos de reproducción sexual y asexual están en las capas más superficiales de los suelos manejados y/o en barbecho (Valbuena \& Trabaud, 2001). En los sistemas agrícolas de cultivos anuales de las zonas tropicales, el manejo de la flora arvense que emerge en ellos constituye uno de los elementos biológicos de mayor importancia que los agricultores deben atender, toda vez que estas plantas originan competencia por los elementos comunes con las plantas cultivadas, además, en algunos casos se presentan efectos alelopáticos, o pueden llegar a ser huéspedes de plagas y enfermedades que afectan a los cultivos (Piudo \& Cavero, 2005).

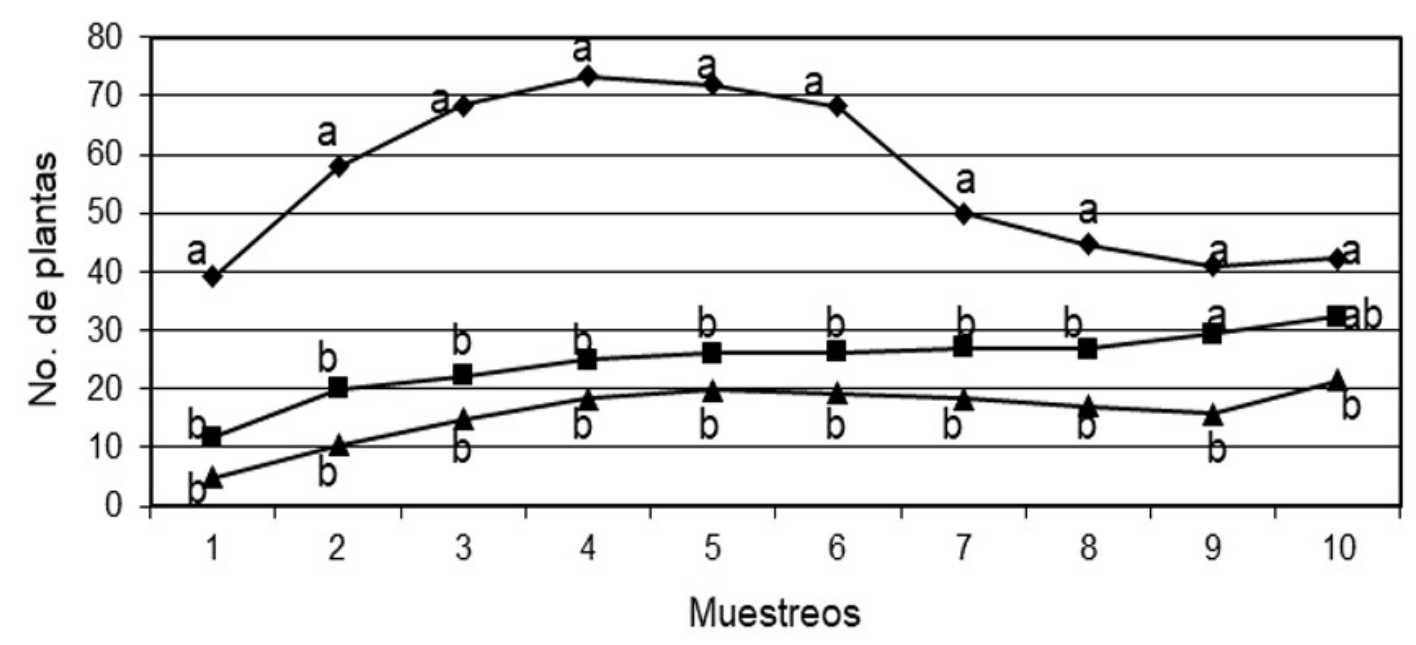

Figura 4. Plantas emergidas a diferentes profundidades.

Diferentes estrategias se han estudiado y puesto en práctica para reducir el efecto negativo del banco de semillas de arvenses presentes en los suelos cultivados, destacándose el uso de herbicidas preemergentes, llamados localmente mata semillas, sin embargo, esto ha llevado a la utilización y dependencia de grandes cantidades de herbicidas sintéticos, que además de causar daños medioambientales y sobre la inocuidad alimentaria, también han promovido la resistencia de las especies de flora endémica sobre los mismos (Aguilar et al., 2020). Este principio de manejo ha traído consigo la utilización de mayores volúmenes de herbicidas con efectos sobre su rentabilidad y sobre la inocuidad de la producción agrícola local. La menor emergencia de plántulas a mayor profundidad está relacionada también con el manejo de los sistemas evaluados. Godoy et al. (1995) encontraron mayor germinación de plántulas a menores profundidades en suelos manejados con labranza convencional, mientras que en sistemas de mínima remoción del suelo la presencia de semillas en las capas su- perficiales del banco fue menor mostrando mayor presencia a mayores profundidades, aludiendo este comportamiento al manejo del suelo.

\subsection{Indicadores biológicos de los bancos de semillas}

En la Tabla 5 se muestran algunos indicadores biológicos de los diferentes reservorios de semillas estudiados. Para número de especies, el terreno de vega, intermedio y el acahual fueron estadísticamente superiores, mientras que para número de individuos en los dos primeros sistemas se presentó el mayor número de especímenes, lo cual es producto del manejo sistemático que se le da al suelo con agricultura de temporada para el cultivo de maíz, lo que origina una dinámica biológica intensa. La riqueza de flora emergente en el sistema de acahual o terreno en barbecho es producto de los procesos de sucesión secundaria en donde la coexistencia de plantas herbáceas, arbustivas y arbóreas aportan de manera sistemática simientes al pool de semillas (Pérez \& Santiago, 2001). 
Tabla 5. Indicadores biológicos del banco de semillas de los sistemas estudiados*

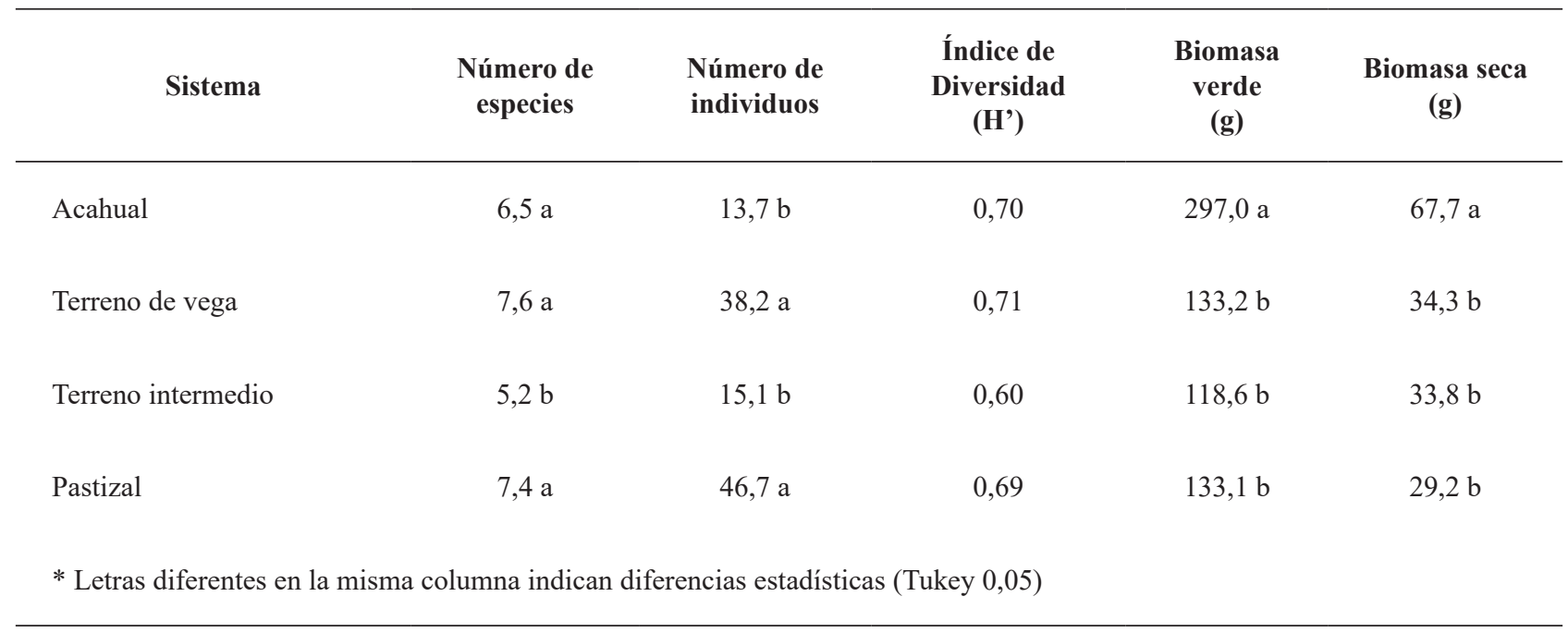

Para la diversidad florística no se encontraron diferencias estadísticas entre los sistemas, lo que nos indica que en el suelo se acumula un número importante de semillas en estado de reposo, las cuales, al existir las condiciones ecológicas adecuadas, fundamentalmente humedad y luz, aprovecharán las circunstancias para iniciar con la colonización de los ecosistemas. Desde la perspectiva del manejo sostenible de los agroecosistemas y de la recuperación de ecosistemas perturbados, la diversidad florística del banco de semillas del suelo constituye un elemento de vital importancia ecológica y socioeconómica, debido a los servicios ambientales presentes y futuros que estos pueden otorgar al sistema ecológico (De Sousa et al., 2006). Pla (2006) concluye que la diversidad cuantificada desde el modelo utilizado será útil cuando se utilice con fines comparativos, ya que se ha demostrado que existen sesgos con la diversidad absoluta cuantificada.

La biomasa total (verde y seca) emergida al final del ensayo en cada una de las charolas de los suelos de los sistemas evaluados, fue superior estadísticamente en el sistema de acahual o barbecho, lo cual refleja que en este sistema germinan fundamentalmente especies arbustivas y arbóreas cuya biomasa es superior a las especies herbáceas (Gordón, 2000). La dinámica biológica establecida en los sistemas estudiados está determinada por el manejo de estos, Bedoya-Patiño et al. (2010) mencionan que las semillas encontradas en los sistemas de sucesión secundaria y de alto grado de perturbación, son más abundantes debido a las múltiples fuentes de propágulos que existen y suceden en ellos como producto de su manipulación sistemática. La biomasa acumulada en los sistemas ecológicos constituye un indicador ambiental de importancia ya que está directamente relacionada con la cantidad de carbono capturado, por ejemplo, Yerema-Yamallel et al. (2014) concluyen que los pastizales en sucesión secundaria retienen una cantidad menor de carbono, en comparación con los matorrales primarios.

\subsection{Indicadores biológicos de las diferentes profundidades}

En la Tabla 6 se muestran los indicadores biológicos por profundidad, observándose para el número de especies y número de individuos una dominancia en las primeras capas de suelo, lo cual nos indica que la acumulación de las semillas en el reservorio de las regiones tropicales se presenta con mayor dinámica en los primeros centímetros. Para el índice de diversidad florística no se encontraron diferencias significativas entre las tres profundidades, lo que nos señala que las plantas emergidas presentan una homogeneidad en la distribución de los individuos dentro de cada una de las especies. Finalmente, para biomasa verde y seca no se determinaron diferencias entre los distintos bancos de semillas, lo que nos indica que una vez que emergen las plantas estas inician su crecimiento y desarrollo independientemente de su tipo y profundidad de localización. Aun cuando las características fisiográficas del territorio y del tipo de suelo influyen en la profundidad de las semillas, en los sistemas perturbados estas estarán presenten en mayor cantidad y diversidad en las primeras capas de suelo (Dalling \& Hubbell, 2002). 
Tabla 6. Indicadores biológicos del banco de semillas a diferentes profundidades*

\begin{tabular}{cccccc}
\hline $\begin{array}{c}\text { Profundidad } \\
(\mathbf{c m})\end{array}$ & $\begin{array}{c}\text { Número de } \\
\text { especies }\end{array}$ & $\begin{array}{c}\text { Número de } \\
\text { individuos }\end{array}$ & $\begin{array}{c}\text { Índice de } \\
\text { Diversidad } \\
\left(\mathbf{H}^{\prime}\right)\end{array}$ & $\begin{array}{c}\text { Biomasa verde } \\
(\mathbf{g})\end{array}$ & $\begin{array}{c}\text { Biomasa } \\
\text { seca } \\
\mathbf{( g )}\end{array}$ \\
\hline $0-10$ & $7,7 \mathrm{a}$ & $38,1 \mathrm{a}$ & 0,72 & 167,2 & 35,3 \\
$10-20$ & $7,2 \mathrm{a}$ & $32,4 \mathrm{a}$ & 0,69 & 207,6 & 48,9 \\
$20-30$ & $5,1 \mathrm{~b}$ & $14,3 \mathrm{~b}$ & 0,62 & 136,7 & 39,6 \\
\hline
\end{tabular}

* Letras diferentes en la misma columna indican diferencias estadísticas (Tukey 0,05)

La importancia del estudio de la dinámica de los bancos de semillas es que estos actúan como filtros evolutivos, determinando los genotipos que pueden sobrevivir bajo condiciones ambientales desfavorables, constituyen la fuente para el reclutamiento de nuevas poblaciones y actúan como almacén de información genética sobre varias generaciones. La variabilidad florística que se da en los bancos de semillas, junto con una fluctuación considerable en la densidad de semillas almacenadas en el suelo a causa de las variables ambientales, provocan cambios en la capacidad de las plantas para su establecimiento (Granados \& López, 2001). Es importante reconocer que, en el pool de semillas del suelo, muchas de ellas están en reposo sin germinar debido al efecto inducido por factores bióticos (inhibición química, periodo de latencia, actividad de microorganismos, etc.) y abióticos (luz, temperatura, humedad, etc.).

\section{5 Índice de similitud de los bancos de semillas}

En cuanto a la similitud entre los diferentes bancos de semillas (Tabla 7), se destaca la analogía superior al $70 \%$ entre el sistema de terreno de vega e intermedio para el cultivo de maíz. Lo anterior es producto del manejo similar de ambos sistemas en donde se cultiva maíz en monocultivo, y donde la estrategia fundamental es el uso de herbicidas sintéticos, los cuales van haciendo selectivas las especies que emergen en los campos cultivados. E1 sistema acahual presentó semejanzas del $30 \%$ con maíz y del $15 \%$ con vega y potrero, indicándonos que las especies presentes en estos bancos están conformadas por especies diferentes a los sistemas agrícolas de manejo intensivo. En el banco de semillas del pastizal se presentó una similitud aproximada del $30 \%$ con los terrenos de cultivo de maíz (vega e intermedio), en donde fundamentalmente las especies de hoja angosta (poáceas) son las que se presentan de forma común en los tres bancos de semillas; resultados similares son reportados por Haretche \& Rodríguez (2006) quienes estudiaron por nueve años el reservorio de semillas de un pastizal y un terreno en descanso, encontrando sucesionalmente mayor similitud en el pastizal y menor en la sucesión secundaria. Por su parte, Gordón (2000) reporta similitudes del $70 \%$ de herbáceas en bancos de semillas de humedales tropicales de sitios diferentes.

Tabla 7. Índice de similitud de los bancos de semilla estudiados

\begin{tabular}{ccccc}
\hline & Acahual & Vega & Maíz & Potrero \\
\hline Acahual & -- & 18,19 & 30 & 15,79 \\
Vega & 18,19 & --- & 72,46 & 32,44 \\
Maíz & 30 & 72,46 & --- & 26,47 \\
Potrero & 15,79 & 32,44 & 26,47 & -- \\
\hline
\end{tabular}




\subsection{Prueba de alelopatía}

Al analizar los efectos alelopáticos de las dos especies arvenses dominantes en los sistemas de cultivo intensivo, terrenos de vega e intermedios de monocultivo con maíz, para número de especies emergidas no se encontró un efecto marcado de coquillo (Cyperus rotundus L.) y flor amarilla (Melampodioum divaricatum Rich.), en ninguna de las concentraciones sobre las tres especies de semillas plantadas (calabaza, frijol y maíz) (Tabla 8).

Tabla 8. Prueba de alelopatía con las arvenses más abundantes*

\begin{tabular}{|c|c|c|c|c|}
\hline Tratamiento & $\begin{array}{c}\text { Concentración } \\
(\%)\end{array}$ & Cultivo & $\begin{array}{l}\text { Número de especies } \\
\text { emergidas }\end{array}$ & $\begin{array}{l}\text { Longitud de la radícula } \\
\qquad(\mathrm{cm})\end{array}$ \\
\hline \multirow{9}{*}{ Flor amarilla } & 2,5 & \multirow{3}{*}{ Calabaza } & 9,33 a & $6,95 \mathrm{abc}$ \\
\hline & & & & \\
\hline & 5,0 & & 9,66 a & $6,67 \mathrm{abc}$ \\
\hline & 2,5 & \multirow{3}{*}{ Frijol } & $10 \mathrm{a}$ & $8,5 \mathrm{ab}$ \\
\hline & & & & \\
\hline & 5,0 & & $8,66 \mathrm{ab}$ & $6,87 \mathrm{abc}$ \\
\hline & 2,5 & \multirow{3}{*}{ Maíz } & $9,66 \mathrm{a}$ & $7,12 \mathrm{abc}$ \\
\hline & & & & \\
\hline & 5,0 & & 9,33 a & $8,11 \mathrm{abc}$ \\
\hline \multirow{9}{*}{ Coquillo } & 2,5 & \multirow{3}{*}{ Calabaza } & $8,33 \mathrm{ab}$ & $8,36 \mathrm{abc}$ \\
\hline & & & & \\
\hline & 5,0 & & $8,33 \mathrm{ab}$ & $7,29 a b c$ \\
\hline & 2,5 & \multirow{3}{*}{ Frijol } & $10 \mathrm{a}$ & $8,76 \mathrm{a}$ \\
\hline & & & & \\
\hline & 5,0 & & $9,33 \mathrm{a}$ & $7,51 \mathrm{bc}$ \\
\hline & 2,5 & \multirow{3}{*}{ Maíz } & 9,33 a & $7,51 \mathrm{abc}$ \\
\hline & & & & \\
\hline & 5,0 & & 9,33 a & $7,14 a b c$ \\
\hline \multirow{9}{*}{ Testigo } & 0 & \multirow{3}{*}{ Calabaza } & $9 \mathrm{ab}$ & $6,90 \mathrm{abc}$ \\
\hline & & & & \\
\hline & 0 & & 9,33 a & $6,96 \mathrm{abc}$ \\
\hline & 0 & \multirow{3}{*}{ Frijol } & $9,33 \mathrm{a}$ & $6,02 \mathrm{c}$ \\
\hline & & & & \\
\hline & 0 & & 9,66 a & $8,02 \mathrm{abc}$ \\
\hline & 0 & \multirow{3}{*}{ Testigo } & $9,66 \mathrm{a}$ & $6,75 \mathrm{abc}$ \\
\hline & & & & \\
\hline & 0 & & $10 \mathrm{a}$ & $6,2 \mathrm{bc}$ \\
\hline
\end{tabular}

* Letras diferentes en la misma columna indican diferencias estadísticas (Tukey 0,05)

Para longitud de la radícula se mostró un patrón similar. Los bajos efectos alelopáticos determinados pueden deberse a una respuesta biológica de las plantas, las cuales, al percibir la presencia de compuestos secundarios (aleloquímicos), responden con un impulso sobre la germinación y crecimiento de su radícula, resultados compartidos por Besnier (1989), mientras que Laynez-Garsaball \& Méndez-Natera (2007) al estudiar el efecto alelopático de la Cyperaceae determinaron que la germinación, altura de 
las plántulas, longitud de las radículas, peso seco del vástago y relación peso seco del vástago/peso seco de la radícula disminuyeron proporcionalmente al aumento de la concentración de extracto, contrariamente, el peso seco de la radícula incrementó, mientras que la relación altura de la plántula/longitud de la radícula no presentó diferencias significativas en las fuentes de variación.

\section{Conclusiones}

La mayor densidad y dominancia relativa de las especies emergidas en el banco de semillas se pre-

\section{Referencias}

Aguilar Jiménez, C. E., Galdámez Gadámez, J., Martínez Aguilar, F. B., Guevara Hernández, F., \& Vázquez Solis, H. (2020). Eficiencia del policultivo maíz-frijol-calabaza bajo manejo orgánico en la Frailesca, Chiapas, México. Revista Cientifica Agroecosistemas, 7(3), 64-72.

Álvarez-Aquino, C., Williams-Linera, G., \& Newton, A. C. (2005). Disturbance Effects on the Seed Bank of Mexican Cloud Forest Fragments. Biotropica, 37(3), 337-342. https://doi. org/10.1111/j.1744-7429.2005.00044.x

Apraez, E., \& Crespo, G., \& Herrera, R. S. (2007). Efecto de la aplicación de abonos orgánicos y mineral en el comportamiento de una pradera de kikuyo (Pennisetum clandestinum Hoechs) en el Departamento de Nariño, Colombia. Revista Cubana de Ciencia Agrícola, 41(1),75-79.

Arbeláez, M. V., \& Parrado-Rosselli, A. (2005). Seed Dispersal Modes of the Sandstone Plateau Vegetation of the Middle Caquetá River Region, Colombian Amazonia. Biotropica, 37(1), 64-72. https://doi.org/10.1111/j.17447429.2005.03077.x

Bakker, J. P. (1989). Nature Management by Grazing and Cutting. On the ecological significance of grazing and cutting regimes applied to restore former species-rich grassland communities in the Netherlands. Dordrecht: Springer. https:// doi.org/10.1007/978-94-009-2255-6 sentaron en los sistemas de terrenos aluviales e intermedios con manejo de monocultivo de maíz, cuantificándose mayor cantidad de especímenes y biomasa de la flora arvense emergida; sin embargo, su diversidad florística fue similar al terreno de pastizal y en sucesión secundaria. La mayor actividad biológica del banco de semillas se presentó en los primeros 10 centímetros del suelo para todos los cuatro sistemas estudiados, reduciéndose de manera significativa a mayores profundidades. La mayor similitud se presentó entre los sistemas de manejo intensivo para cultivo de maíz, reduciéndose en comparación con el pastizal y muy significativamente con el sistema de acahual o en sucesión secundaria.

Bedoya-Patiño, J. G., Estévez-Varón, J. V., \& Castaño-Villa, G. J. (2010). Banco de semillas del suelo y su papel en la recuperación de los bosques tropicales. Boletín Científico del Centro de Museos, 14(2), 77-91.

Besnier, R. F. (1989). Semillas: Biología y tecnología. Madrid: Ediciones Mundi Prensa.

Cantillo, H. E. E., Castiblanco, G. V., Pinilla, M., D. F., \& Alvarado, C. L. (2008). Caracterización y valoración del potencial de regeneración del banco de semillas germinable de la reserva forestal Cárpatos (Guasca, Cundinamarca). Colombia Forestal, 11(1), 45-70.

Cubiña, A., \& Aide, T. M. (2001). The effect of distance from forest edge on seed rain and soil seed bank in a tropical pasture. Biotropica, 33(2), 260-267. https://doi.org/10.1111/j.1744-7429.2001.tb00177.x

Dalling, J. W., \& Hubbell S. P. (2002). Seed size, growth rate and gap microsite conditions as determinants of recruitment success for pioneer species. Journal of Ecology, 90(3), 557-568.https://doi.org/10.1046/j.13652745.2002.00695.x

De Souza Maia, M., Maia, F., \& Pérez, M. (2006). Bancos de semillas en el suelo. AgriScientia, 23(1), 33-44. https://doi.org/10.31047/1668.298x.v23.n1.2689

Fenner, M., \& Thompson, K. (2005). The ecology of seeds. Cambridge: Cambridge University Press. 
García. (1987). Modificaciones al sistema de clasificación climática de Köppen. (4a ed.). México: Talleres de offset Larios.

Gliessman, S. R. (2002). Agroecología: procesos ecológicos en la agricultura sostenible. Costa Rica: CATIE.

Godoy, G., Vega, J., \& Pitty, A. (1995). El tipo de labranza afecta la flora y la distribución vertical del banco de semillas de malezas. CEIBA, $36(2), 217-229$.

Gordón, E. (2000). Dinámica de la vegetación y del banco de semillas en un humedal herbáceo lacustrino (Venezuela). Revista de Biología Tropical, 48(1), 25-42.

Granados, S. D., \& López, R. G. F. (2001). Ecología de poblaciones vegetales. México: Universidad Autónoma Chapingo.

Granados, S. D., López, R. G., \& Gama, F. (2001). Interacciones ecológicas de las plantas. Texcoco, México: Universidad Autónoma Chapingo.

Gutiérrez, C. J. G., Aguilera, G. L. I., \& González, E. C. E. (2008). Agroecología y sustentabilidad. Convergencia, 15(46), 51-87.

Haretche, F., \& Rodríguez, C. (2006). Banco de semillas de un pastizal uruguayo bajo diferentes condiciones de pastoreo. Ecología Austral, 16, 105-113.

Laynez-Garsaball, J. A., \& Méndez-Natera J. R. (2007). Efectos de extractos acuosos de la maleza Cyperus rotundus L. (Cyperaceae) sobre la germinación de semillas y crecimiento de plántulas de maíz (Zea mays L.) cv. Pioneer 3031. Revista Peruana de Biología, 14(1), 055-060.

León, R., \& Agüero, R. (2001). Efectos de tipos de labranza sobre la población de malezas en caña de azúcar. Agronomía Mesoamericana, 12(1), 71-78.

López Báez, W., Reynoso Santos, R., López Martínez, J., Camas Gómez, R.,\& Tasistro, A. (2018). Diagnóstico de la compactación en suelos cultivados con maíz en la Región Fraylesca, Chiapas. Revista Mexicana de Ciencias Agrícolas, 9(1), 65-79. http://dx.doi.org/10.29312/ remexca.v9i1.848
López Báez, W., Reynoso Santos, R., López Martínez, J., Villar Sánchez, B., Camas Gómez, R., \& García Santiago, J. (2019). Caracterización físico-química de suelos cultivados con maíz en Villaflores, Chiapas. Revista Mexicana de Ciencias Agricolas, 10(4), 897-910. https://doi. org/10.29312/remexca.v10i4.1764

López-Báez W., Reynoso-Santos R., Camas Gómez R., \& Santos-Clemente E. C. (2019). Caracterización de los suelos cultivados con café $(\mathrm{Co}$ $f f e a \mathrm{~L}$ ) en la Sierra Madre de Chiapas, México. Agroproductividad, 12(1), 53-58. https://doi. org/10.32854/agrop.v0i0.1338

Martínez, F. B., Guevara, F., Aguilar, C. E., Pinto, R., La O, M. A., Rodríguez, L. A., \& Aryal, D. R. (2020). Energy and economic efficiency of maize agroecosystem under three management strategies in the Frailesca, Chiapas (México). Agriculture, 10(3), 81. https://doi.org/10.3390/ agriculture 10030081

Moscoso, M. L. B., \& Diez, G. M. C. (2005). Banco de semillas en un bosque de roble de la Cordillera Central Colombiana. Revista Facultad Nacional de Agronomía Medellín, 58(2), 29312943.

Pérez, E. M., \& Santiago, E. T. (2001). Dinámica estacional del banco de semillas en una sabana de los Llanos Centro Orientales de Venezuela. Biotrópica, 33(3), 435-446. https://doi.org/10.1111/j.1744-7429.2001.tb00197.x

Piudo, M. J., \& Cavero, R. Y. (2005). Banco de semillas: Comparación de metodologías de extracción, de densidad y de profundidad de muestreo. Publicaciones de Biología, Universidad de Navarra, Serie Botánica, 16, 71-85.

Pla, L. (2006). Biodiversidad: inferencia basada en el índice de Shannon y la riqueza. Interciencia: Revista de ciencia y tecnología de América, 31(8), 583-890.

Price, M. V., \& Joyner, J. W. (1997). What resources are available to desert granivores: seed rain and soil seed bank? Ecology, 78(3), 764-773. https://doi.org/10.2307/2266056

Sans, F. X. (2007). La diversidad de los agroecosistemas. Ecosistemas, 16(1), 44-49. https://doi. org/10.7818/re.2014.16-1.00 
Shannon, C. E., \& Wiener, W. (1949). The Mathematical Theory of Communication. Illinois: University Illinois Press, Urbana.

Sørensen, T. (1957). A method of establishing groups of equal amplitude in plant sociology based on similarity of species and its application to analyses of the vegetation on Danish commons». Kongelige Danske Videnskabernes Selskab, 5(4), 1-34.

Thompson, K., \& Grime, J.P. (1979). Seasonal variation in the seed banks of herbaceous species in ten contrasting habitats. Journal of Ecology, 67(3), 893-921. https://doi. org/10.2307/2259220

Valbuena, 1., \& Trabaud, L. (2001). Contribution of the soil seed bank to post-fire recovery of a heathland. Plant Ecology, 152, 175-183. https:// doi.org/10.1023/A:1011419721307
Walck, J., Baskin, J., Baskin, C., \& Hidayati, S. (2005). Defining transient and persistent seed banks in species with pronounced seasonal dormancy and germination patterns. Seed Science Reseach, 15(3), 189-196. https://doi. org/10.1079/SSR2005209

Yerena Yamallel, J. I., Jiménez Pérez, J., Alanís Rodríguez, E., Aguirre Calderón, O. A., González Tagle, M. A., \& Treviño Garza, E. J. (2014). Dinámica de la captura de carbono en pastizales abandonados del noreste de México. Tropical and Subtropical Agroecosystems, 17(1), 113-121.

Yu, S., Sternberg, M., Kutiel, P., \& Chen, H. (2007). Seed mass, shape, and persistence in the soil seed bank of Israeli coastal sand dune flora. Evolutionary Ecology Research, 9, 325-340. 